\title{
Relación entre las características antropométricas de masa muscular de extremidad inferior y la potencia de salto de jóvenes deportistas
}

Benavides-Roca, Luis; Salazar Orellana, Cristian; Díaz Coria, Gastón

Relación entre las características antropométricas de masa muscular de extremidad inferior y la potencia de salto de jóvenes deportistas

MHSalud, vol. 18, núm. 2, 2021

Universidad Nacional, Costa Rica

Disponible en: https://www.redalyc.org/articulo.oa?id=237066090009

DOl: https://doi.org/10.15359/mhs.18-2.9

\section{(c) (1) (9)}

Esta obra está bajo una Licencia Creative Commons Atribución-NoComercial-SinDerivar 3.0 Internacional. 


\section{Relación entre las características antropométricas de masa muscular de extremidad inferior y la potencia de salto de jóvenes deportistas} Relation Between Anthropometric Muscle Mass Characteristics of Lower Limbs and Jump Power in Young
Athletes

Relação entre as características antropométricas da massa muscular dos membros inferiores e a força de salto de jovens atletas

Luis Benavides-Roca

Universidad Santo Tomas, Escuela Ciencias del Deporte,

Chile

DOI: https://doi.org/10.15359/mhs.18-2.9

Redalyc: https://www.redalyc.org/articulo.oa?

Benavides.roca@gmail.com

(iD https://orcid.org/0000-0002-0995-2564

Cristian Salazar Orellana

Universidad Santo Tomas, Escuela Ciencias del Deporte,

Chile

cristiansalazaror@santotomas.cl

(iD) https://orcid.org/0000-0002-3431-3203

Gastón Díaz Coria

Universidad Católica del Maule, Chile

gasdico@yahoo.com

(iD https://orcid.org/0000-0002-3260-3071

Recepción: 28 Agosto 2020

Aprobación: 07 Abril 2021

\section{Resumen:}

La antropometría regional resulta ser un procedimiento importante en la proyección del desempeño de un deportista. El objetivo del presente estudio es relacionar las características antropométricas de extremidad inferior con la potencia de salto en jóvenes deportistas. Se utilizó una metodología correlacional donde se evaluaron a 44 deportistas juveniles (30 hombres y 14 mujeres), a los cuales, se les midió la altura del salto en sentadilla de $90^{\circ}$ (SJ), contra movimiento con manos fijas (CMJ-MF) y contra movimiento con manos libres (CMJ-ML). Estas variables derivaron al cálculo de potencia. Junto con esto se determinó características antropométricas de miembro inferior vinculados a pliegues cutáneos y perímetros, obteniendo valores de muslo medio (MMED) y máximo (MMAX), perímetro corregido de pantorrilla (PCP), área de sección muscular de cuádriceps (ASMC), área de sección muscular de isquiotibial (ASMI) y área de sección muscular total de muslo (ASMM). Los resultados mostraron una correlación significativa entre el perímetro corregido de pantorrilla y la potencia de salto en $\mathrm{SJ}(r=0,73 ; p=0,00)$, CMJ-MF $(r=0,72 ; p=0,00)$ y CMJ-ML $(r=0,7 ; p=0,00)$. En conclusión, el perímetro de pantorrilla presentó componentes relacionados a la potencia de salto de los deportistas juveniles.

Palabras claVe: antropometría, potencia, salto, deportistas.

\section{Abstract:}

The regional anthropometry turns out to be an important procedure in projection of the athlete's performance. The objective of the present study is to relate the anthropometric characteristics of the lower limbs of the body with the jumping power of young athletes. It was used a correlational methodology where 44 youth athletes ( 30 men and 14 women) were evaluated. Which ones calculated a $90^{\circ}$ squat jump height against movement (CMJ-MF) and free, these variables derived to calculate the power. With this we determinate the anthropometric characteristic of lower body parts linked to skinfolds and perimeters, obtaining values of midthigh (MMED) and maximum (MMAX), corrected calf perimeter (PCP), quadriceps muscle section area (ASMC), hamstring muscular section area(ASMI)and total section muscular thigh area(ASMM) The results showed a significant correlation between the corrected calf perimeter and the jump power in SJ $(r=0.73 ; p=0.00)$, CMJ-MF $(r=0.72 ; p=0.00)$ and free $(r=0.7 ; p=$ $0.00)$. In conclusion, the calf perimeter presented related components to the young's athletes jumping power. 
KEYWORDS: anthropometry, power, jump, athletes.

\section{Resumo:}

A antropometria regional acaba sendo um procedimento importante na projeção do desempenho de um atleta. O objetivo do presente estudo é relacionar as características antropométricas do membro inferior com a força de salto em jovens atletas. Foi utilizada uma metodologia correlacional onde foram avaliados 44 atletas jovens ( 30 homens e 14 mulheres), para os quais foi medida a altura do salto no agachamento a $90^{\circ}(\mathrm{SJ})$, contra movimento com mãos fixas (CMJ-MF) e movimento contrário livre (CMJ-ML). Essas variáveis levaram ao cálculo da potência. Paralelamente, foram determinadas as características antropométricas do membro inferior relacionadas às dobras cutâneas e perímetros, obtendo-se os valores da coxa média (MMED) e máxima (MMAX), perímetro corrigido da panturrilha (PCP), área de seção muscular do quadríceps (ASMC), área da seção do músculo isquiotibial (ASMI) e área total da seção do músculo da coxa (ASMM). Os resultados mostraram uma correlação significativa entre a circunferência da panturrilha corrigida e a força de salto em SJ $(r=0,73 ; p=0,00)$, CMJ-MF $(r=0,72 ; p=0,00)$ e CMJ$\operatorname{ML}(r=0,7 ; p=0,00)$ Em conclusão, a circunferência da panturrilha apresentou componentes relacionados à força de salto dos jovens atletas.

Palavras-CHAVE: antropometria, potência, salto, atletas.

\section{INTRODUCCIÓN}

El desarrollo de la vida, conlleva al ser humano a realizar constantes movimientos que en su esencia son provocados por la generación de fuerza, es por ello, que resulta imprescindible generar adaptaciones que conecten el cuerpo con el medio en el que se desenvuelve (Pereira et al., 2018).

En torno a la competición, el rendimiento del atleta requiere de muchos factores que provoquen un beneficio en su nivel deportivo, lo que, a su vez, está influenciado por la modalidad, los requerimientos o la singularidad de cada disciplina. Lo anterior, tiene relación con el desarrollo de las capacidades físicas, esencialmente con la fuerza, la cual, está presente en cada acción técnica del deporte, a partir de esto, se desprenden capacidades subyacentes como la potencia, la que es entendida, como la resultante de un movimiento generado por fuerzas a una determinada velocidad.

Particularmente, podemos encontrar situaciones donde se utilizan potencias máximas o medias, lo que va a estar determinado por las necesidades específicas del deporte, por ejemplo, el salto resulta ser una acción que conlleva el desplazamiento del cuerpo en función de un objetivo, más aún, este movimiento puede ser descrito como un proceso neurofisiológico, donde interactúan unidades motoras, las cuales, se reclutan para la contracción muscular, provocando un acortamiento de la fibra, dado por la ordenanza del impulso nervioso (Suchomel et al., 2018).

A partir de esto, se encuentra el ciclo estiramiento acortamiento, el que hace referencia a la capacidad del músculo de realizar este proceso durante una acción dinámica de alta velocidad, lo que va a estimular la producción de la potencia, debido a la utilización de la energía elástica, acumulada en las fibras musculares durante la segunda fase de un salto en contra movimiento (Hatfield et al., 2006).

Por tanto, se hace esencial la presencia de la máxima potencia mecánica, la cual, es posible de evidenciar a través de los test de salto, donde la musculatura del tren inferior tiene la mayor implicancia (Jiménez et al., 2009). Además, esta prueba presenta una gran transversalidad en términos de evaluación, abarcando el área de la salud y del deporte, lo que la hace apta para todo tipo de personas y óptima para el análisis de un especialista (Oteo et al., 2015).

El test de salto, es un procedimiento muy importante para la medición de las capacidades físicas en los deportes que se asocian en mayor medida con la fuerza (Castagna et al., 2013). Específicamente, las elevaciones verticales son usadas por los entrenadores para evaluar la potencia muscular del impulso provocado por el cuerpo (Jiménez-Reyes y González-Badillo 2011). 
Particularmente, la medición del salto en sentadilla de $90^{\circ}$ (SJ) es un contraste de la fuerza explosiva, el salto en contra movimiento con manos fijas (CMJ-MF) representa la fuerza elástica explosiva y el salto libre (CMJ-ML) una adecuación a la técnica propia de la disciplina.

Por su parte, estas evaluaciones permiten obtener variables asociadas al índice elástico, pliométrico, coordinativo general y de reactividad, los cuales, son datos a considerar para la planificación del entrenamiento y el nivel de desempeño del deportista (Bosco et al., 1983; Di Giminiani y Scrimaglio, 2006).

Las características propias del salto, hacen entender la eficiencia del atleta, ya que, tanto en deportes colectivos como individuales, existen acciones que se vinculan con la capacidad de elevarse, por ejemplo, las acrobacias, la generación de fuerzas excéntricas y hasta movimientos preventivos de lesiones.

Lo anterior, propicia el desarrollo físico de un deportista para los procesos competitivos a los cuales se somete (Ferragut y López, 1998; Raya-González et al., 2018; Quiroga Maraboli et al., 2016). Esta evidencia hace entender que la evaluación de salto debe estar presente en los diversos deportes, para el seguimiento de la planificación del entrenamiento.

Por su parte, la valoración antropométrica es un procedimiento accesible a la hora de controlar características de un deportista, es por ello, que resulta de interés determinar la influencia que tiene sobre los componentes del área de la actividad física (Al-Gindan et al., 2014), por ejemplo, cuantificar la masa adiposa y muscular, permite proyectar un buen rendimiento mecánico, estimar la producción de fuerza y el trabajo, también, se relaciona con el coste metabólico, los efectos anabólicos del entrenamiento y el rendimiento muscular (Berral de la Rosa et al., 2010).

Además de esto, la mayoría de los procesos de entrenamiento converge en el modelo de la hipertrofia, donde se relaciona el tamaño de la masa muscular con la fuerza, ya que, a mayor masa va existir una mejor estimulación de los componentes específicos de los músculos, como son las miofibrillas y el sarcoplasma, lo cual, puede ser reproducible en una mayor potencia (Reggiani, \& Schiaffino, 2020)

Se tiene en cuenta que la composición corporal es capaz de afectar el desempeño de los deportistas, ya que, algunas características antropométricas producen niveles altos o bajos de ejecución, por ejemplo, en el vóleibol los sujetos presentan mayor masa muscular, menor grasa y más longitud en sus estructuras, en comparación con una persona que no practica este deporte (González-Ravé et al., 2011).

Por tanto, la relación de los aspectos antropométricos con el desarrollo del salto en los deportes, se puede relacionar con el balance de la masa muscular (Taylor et al., 2010) y la masa grasa (Rendón et al., 2017). Si bien, la medición de las características corporales es utilizada constantemente en el mundo deportivo, resalta la idea de asociar aspectos regionales del cuerpo con atributos físicos, ya que, las singularidades de las disciplinas conllevan a entender componentes específicos del cuerpo con determinados movimientos deportivos.

Por lo cual, el objetivo de esta investigación es determinar la relación de la antropometría regional de miembro inferior con la potencia de salto en deportistas juveniles, proyectando como hipótesis la correlación positiva entre la potencia de salto, con alguna de las variables antropométricas de miembro inferior.

\section{Metodología}

El presente estudio tiene un diseño de investigación correlacional trasversal, que permite ver la interacción entre las variables de potencia de salto y las características antropométricas de miembro inferior.

\section{Participantes}

Fueron evaluados 44 sujetos, de los cuales eran 30 hombres y 14 mujeres del programa de

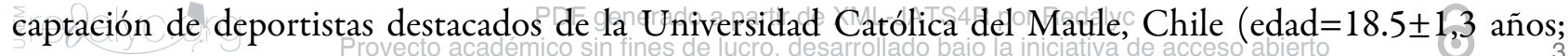


peso $=71.5 \pm 11,4 \mathrm{~kg} ;$ talla $=1.72 \pm 0,1 \mathrm{~m}$; años de entrenamiento $=2.8 \pm 0.5$ años), los cuales pertenecían a las especialidades de fútbol, voleibol, baloncesto, balón mano, atletismo y gimnasia. Si bien, las características deportivas, son distinta en cada una de estas disciplinas, la potencia resulta ser un componente unificador en todas ellas, debido a las acciones determinantes del éxito en cada uno de estos deportes, como son los saltos, el sprint o un cambio de dirección.

El muestreo utilizado fue de tipo no probabilístico por conveniencia, donde existió en primera instancia una revisión de los antecedentes deportivos del sujeto, para luego ser seleccionado para el proceso de evaluaciones físicas.

Dentro de los protocolos de la Universidad Católica del Maule los atletas debían firmar un consentimiento informado, donde se especificaba las características de las evaluaciones y los componentes éticos del tratamiento de los datos. Lo anterior estaba basado en el tratado de Helsinki y las normativas éticas de la propia institución.

\section{Instrumentos}

Las medidas antropométricas fueron realizadas por personas con experiencia en la cineantropometría y con certificación International Society for the Advancement of Kinanthropometry nivel III. Para la medida de peso se utilizó una balanza marca Tanita Modelo HD 314, Japón, con una escala de medición de 0 a $150 \mathrm{~kg}$.

Para la talla se empleó un estadiómetro marca Seca 750, Hamburg, con una precisión de $1 \mathrm{~mm}$; el porcentaje de grasa, fue obtenido con un plicómetro marca Harpenden, West Sussex, Reino Unido, con una precisión de $0.2 \mathrm{~mm}$ y para la medición de los perímetros se utilizó una cinta métrica de $1 \mathrm{~mm}$ de precisión.

Por su parte, las evaluaciones de potencia fueron ejecutadas con la plataforma de contacto DMJUMP marca Prometheus Sportech ${ }^{\circledR}$, la que está conectada a un software DMJ V2.2 Beta, permitiendo contrastar datos a partir de la relación de tiempo de vuelo con la altura de salto. Este instrumento fue validado en Chile por Saavedra y Vergara (2013).

\section{Procedimiento}

Las evaluaciones antropométricas siguieron los protocolos establecidos por la ISAK (Marfell-Jones et al., 2012), la cual, establece marcaje y medición de variables: masa corporal, talla, perímetro de muslo máximo, medio y pantorrilla y el porcentaje de grasa de muslo y pantorrilla. Los sujetos, se evaluaron durante el periodo de la mañana sin haber realizado alguna actividad previa, para la evaluación de la masa corporal ( $\mathrm{kg}$ ) se midieron sin calzado y con la menor cantidad de ropa posible, la estatura fue medida en el plano de Frankfurt sin zapatos.

A partir de los datos obtenidos y utilizando las ecuaciones de Ross y Kerr (1991) y Housh et al. (1995), fue posible determinar las variables de perímetro corregido de muslo (máximo y medio), pantorrilla y área de sección muscular de cuádriceps, muslo e isquiotibiales respectivamente (Ver tabla 1).

TABLA 1

\section{Ecuaciones antropométricas de miembro inferior}

\begin{tabular}{ccc}
\hline $\begin{array}{c}\text { Ross y Kerr } \\
(1991)\end{array}$ & $\begin{array}{c}\text { Perímetro de muslo medio corregido }(\mathrm{cm}) \\
\text { Perímetro de muslo máximo corregido }(\mathrm{cm})\end{array}$ & $\begin{array}{c}\text { PM Medio- }(\mathrm{PAM}(\mathrm{cm}) * \pi \\
\text { PM Máximo- }(\mathrm{PAM}(\mathrm{cm}) * \pi\end{array}$ \\
\hline & Perímetro de pantorrilla corregido $(\mathrm{cm})$ & PP- $(\mathrm{PPM}(\mathrm{cm}) * \pi$ \\
\hline $\begin{array}{c}\text { Housh et al. } \\
(1995)\end{array}$ & Área de sección muscular de cuádriceps $\left(\mathrm{cm}^{2}\right)$ & $(2.52 * \mathrm{PM})-(1.25 * \mathrm{PAM})-45.13$ \\
& Área de sección muscular de isquiotibiales $\left(\mathrm{cm}^{2}\right)$ & $(1.98 *$ PM $)-(0.64 * \mathrm{PAM})-22.69$ \\
& Área de sección muscular total de muslo $\left(\mathrm{cm}^{2}\right)$ & $(4.68 * \mathrm{PM})-(2.09 * \mathrm{PAM})-80.99$ \\
\hline
\end{tabular}

$\pi=\mathrm{Pi}(3,1416), \mathrm{PM}=$ perímetro de muslo $(\mathrm{cm}),. \mathrm{PAM}=$ pliegue anterior del muslo

$(\mathrm{mm})$, PP: perímetro de pantorrilla $(\mathrm{cm})$, PPM: pliegue de pierna medial $(\mathrm{mm})$. 
Para determinar los valores de potencia, se utilizó la altura de salto, a partir de la metodología propuesta por Bosco (1994), en SJ, CMJ-MF y CMJ-ML, los cueles derivaron a las fórmulas expuestas por Sayers et al. (1999) y Lara et al. (2004), donde se obtuvo la potencia. El protocolo de la evaluación de salto indica que el SJ se ejecuta con una flexión de $90^{\circ}$ con pies paralelos y manos en la cadera (Ver tabla 2).

Por su parte el CMJ-MF corresponde a un salto en el cual, el sujeto comienza en bipedestación con las manos en cadera, luego viene una flexión de rodilla de $90^{\circ}$, para finalmente, ejecutar la elevación del cuerpo; por su parte, el salto CMJ-ML tiene la característica de ser ejecutado de la manera más cómoda posible, con la indicación de asemejarlo algún gesto técnico de su deporte.

Previo a los test, los deportistas realizaron un calentamiento de $10 \mathrm{~min}$. donde se involucraban movimientos asociados a la medición y acciones explosivas que derivaban a la potencia (sprint, caídas, cambios de dirección, etc.) para finalizar, se les recomendaba elongar de manera balística esperando su turno. También cabe señalar, que los deportistas poseían alguna familiarización con los test, por lo cual, se les solicitó realizar dos intentos contabilizando el de mejor desempeño. El tiempo entre cada evaluación fue de aproximadamente de 3 minutos.

TABLA 2

Ecuaciones de potencia (w)

\begin{tabular}{cccc}
\hline \multirow{2}{*}{ Sayer et al. } & SJ & Potencia (w) & $(51.9 *$ altura CMJ-MF $(\mathrm{cm}))+\left(48.9 * \mathrm{MC}_{(\mathrm{kg})}\right)-2007$ \\
& CMJ-MF & Potencia (w) & $\left(60.7 *\right.$ altura SJ $\left.\mathrm{Scm}_{(\mathrm{cm})}\right)+\left(45.3 * \mathrm{MC}_{(\mathrm{kg})}\right)-2056$ \\
\hline Lara et al. & CMJ-ML & Potencia (w) & $(62.5 *$ altura de salto $(\mathrm{cm}))+\left(50.3 * \mathrm{MC}_{(\mathrm{kg})}\right)-2184.7$ \\
\hline
\end{tabular}

$\mathrm{MC}=$ Masa corporal $(\mathrm{kg}), \mathrm{SJ}=$ Salto desde flexión $90^{\circ}$ de rodilla, $\mathrm{CMJ}-\mathrm{MF}=$ Salto con contra movimiento

Tanto las evaluaciones antropométricas como las de salto, son parte del protocolo de ingreso a la Universidad Católica del Maule, por tanto, todas las mediciones fueron efectuadas en las dependencias del establecimiento.

\section{Análisis de datos}

El análisis estadístico se realizó en el programa SPSS Statiscs 22. Los datos se sometieron a la prueba de normalidad Kolmogorov-Smirnov y se calcularon estadísticos descriptivos de media y desviación estándar considerando un intervalo de confianza del $95 \%$. Se realizó la prueba de correlación de Pearson donde los índices de hasta " 0.39 ” se consideraron como una correlación débil, entre " 0.40 a 0.69 ” correlación moderada, de “ 0.70 a 0.89 ” como una correlación fuerte, mientras que “ $\geq 0.9$ ” se considerará una correlación muy fuerte (Schober et al., 2018).

\section{Resultados}

A continuación, se muestran los resultados obtenidos a partir de la medición de las variables antropométricas y la capacidad de salto de los deportistas preuniversitarios de la Universidad Católica del Maule. 
MHSALUD, ISSN: 1659-097X, 18(2), Julio-Diciembre, 2021, PP 1-11

Benavides-Roca, Salazar Orellana, Díaz Coria

TABLA 3

Antropometría de miembro inferior y rendimiento de la capacidad de salto

\begin{tabular}{|c|c|c|c|c|c|c|c|c|}
\hline \multicolumn{3}{|c|}{ Potencia (w) } & \multicolumn{3}{|c|}{ Perímetros (cm) } & \multicolumn{3}{|c|}{ Área (cm2) } \\
\hline Sayer ss & Sayer CмJ-MF & Lara CMJ-ML & MMED & MMAX & PCP & ASMC & ASMI & ASMM \\
\hline $2959 \pm 731.2$ & $3128.2 \pm 754.2$ & $3735.3 \pm 894.4$ & $51.6 \pm 4$ & $53.8 \pm 4$ & $32.7 \pm 2.6$ & $85.1 \pm 10.1$ & $83.6 \pm 7.9$ & $163.5 \pm 18.6$ \\
\hline
\end{tabular}

Sayer SJ: cálculo de potencia del Sj; Sayer CMJ-MF: cálculo de potencia del CMJ-MF; Lara CMJ-ML: cálculo de potencia del salto CMJ-ML; MMED: muslo medio; MMAX: muslo máximo, PCP: perímetro corregido de pantorrilla; ASMC: área de sección muscular de cuádriceps, ASMI: área de sección muscular isquiotibial; ASMM: Área de sección muscular total de muslo.

La tabla 3, presenta que el muslo posee mayores valores en comparación con el perímetro de pantorrilla y que el área muscular de cuádriceps $(85.1 \pm 10.1 \mathrm{~cm} 2)$ es mayor a la de isquiotibial $(83.6 \pm 7.9 \mathrm{~cm})$. Por su parte, la potencia de salto muestra que los sujetos alcanzan mejor desempeño en el salto CMJ-ML (3735.3 $\pm 894.4 \mathrm{w})$.

TABLA 4

Correlación de la antropometría y la potencia de miembro inferior

\begin{tabular}{lccccccccc}
\hline & & ASMM & PC_MMED & PC_MMAX & PCP & ASMC & ASMI & PESO & TALLA \\
\hline Sayer SJ & $r$ & 0.46 & 0.57 & 0.45 & $0.73^{*}$ & 0.46 & 0.45 & $0.64 *$ & $0.71 *$ \\
& $p$ & 0.00 & 0.00 & 0.00 & 0.00 & 0.00 & 0.00 & 0.00 & 0.00 \\
Sayer & $r$ & 0.48 & 0.57 & 0.47 & $0.72 *$ & 0.49 & 0.47 & $0.79 *$ & $0.73^{*}$ \\
CMJ-MF & $p$ & 0.00 & 0.00 & 0.00 & 0.00 & 0.00 & 0.00 & 0.00 & 0.00 \\
Lara & $r$ & 0.39 & 0.5 & 0.37 & $0.7 *$ & 0.39 & 0.37 & $0.71 *$ & $0.7 *$ \\
CMJ-ML & $p$ & 0.01 & 0.00 & 0.01 & 0.00 & 0.01 & 0.01 & 0.00 & 0.00 \\
\hline
\end{tabular}

${ }^{*} \mathrm{r} \geq 0.7 \mathrm{y}$ con un $\mathrm{p}<0.05$

La tabla 4, muestra la correlación de las características antropométricas de miembro inferior y la potencia de salto. Se observa que la variable de diámetro de pantorrilla (SJ: $r=0.73, p=0.00$; CMJ-MF: $r=0.72, p$ $=0.00$; Lara: $r=0.7, p=0.00$ ), peso (SJ: $r=0.64, p=0.00$; CMJ-MF: $r=0.79, p=0.00$; Lara: $r=0.71$ $p=0.00)$ y talla (SJ: $r=0.71, p=0.00$; CMJ-MF: $r=0.73, p=0.00$; Lara: $r=0.7, p=0.00)$ presenta una relación significativa con todos los datos de potencia.

\section{Discusión}

Si bien los estudios de antropometría y rendimiento físico, ya han sido bastante estudiados, la influencia de regiones específicas del cuerpo pareciera ser un área a explorar, es por ello, que el objetivo del presente estudio es analizar las características antropométricas del miembro inferior con la potencia de salto de deportistas juveniles.

De manera específica en el estudio de Sheppard et al. (2008) se evaluó voleibolistas, relacionando aspectos antropométricos y capacidad de salto, encontrando correlaciones significativas entre el CMJ-MF y la estatura de los deportistas $(r=0.77 ; p=0.00)$, de igual forma que los sujetos del presente estudio.

En torno al desempeño de la altura alcanzada en los saltos, se observa que los participantes del presente estudio, manifiestan un rendimiento inferior a grupos de deportistas de combate, tanto en el SJ (34.2 \pm 6.9 $\mathrm{cm})$ como CMJ-MF $(37.1 \pm 7.8 \mathrm{~cm}$ ) (Spigolon et al., 2018), de distinta forma, al comparar los resultados con un grupo de la categoría sub 20 de hockey patín $(S J=27.4 \pm 4.6 \mathrm{~cm}$; CMJ-MF $=30.4 \pm 4.4 \mathrm{~cm}$ ), se muestran 
un rendimiento superior por parte de los atletas juveniles de la Universidad Católica del Maule (Ferreira et al., 2019).

De acuerdo a Markovic et al. (2004), el CMJ-MF es el mejor indicador de la potencia muscular en las extremidades inferiores y además resulta ser un gran predictor del rendimiento en pruebas de velocidad durante la semana de competición (Jiménez-Reyes y González-Badillo, 2011).

Al comparar al grupo de deportistas juveniles en el CMJ-MF, con sujetos que practican yudo (43.3 \pm 7.0 $\mathrm{cm}$ ) y jiu jitsu $(45.3 \pm 4.1 \mathrm{~cm})$ presentan un menor rendimiento (Lima-Kons et al., 2017), y sucede lo mismo, al contrastar los resultados con De Campos, Leporce y Souto-Maior (2019), los que evaluaron a futbolistas $(37.7 \pm 7.4 \mathrm{~cm})$ y peleadores de artes marciales mixtas $(41.6 \pm 6.1 \mathrm{~cm})$.

Este hallazgo muestra un mejor desempeño en los deportes de combate, con respectos a las disciplinas de este estudio, lo cual, puede ser debido al rol que tienen las extremidades inferiores en el desarrollo y éxito del deporte, por ejemplo, patadas, rebotes y bloqueos, son asociados a movimiento explosivos y con altos niveles de fuerza, vinculados a la eficiencia del ciclo estiramiento acortamiento (Ojeda-Aravena et al., 2019).

En torno a las características antropométricas, se entiende que el porcentaje de grasa al estar presente de manera significativa en la región inferior del cuerpo genera una influencia negativa del desempeño de los atletas en el salto vertical o una acción motora (Apostolidis et al., 2004; Moreira et al., 2008).

Es por ello, que Ozbar, Ates y Agopyan (2014), proponen la implementación del entrenamiento de fuerza pliométrico durante 8 semanas, con un volumen progresivo de 90 hasta 220 saltos, como una opción adecuada para generar adaptaciones, en la disminución del porcentaje de grasa total y el aumento del desempeño de potencia (Pre=3.480,0 $\pm 6643,2$ Post $=3.855,2 \pm 6536,6)$.

En torno a la literatura, se puede recabar que existe una relación importante entre los beneficios de poseer masa muscular y tener un alto desempeño deportivo (Pasin et al., 2017). Se entiende que la antropometría es una poderosa variable de medición en el ámbito deportivo, por lo cual, es necesaria la implementación de esta técnica, para la búsqueda del alto rendimiento (Rodríguez et al., 2010).

Investigaciones relacionadas a lo antropométrico y al desarrollo físico, muestran que los diámetros y porcentajes bajos de masa grasa de determinadas partes del cuerpo se asocian a niveles elevados de potencia tanto en deportes individuales como colectivos (Temfemo et al., 2009; Spigolon et al., 2018), de igual forma que los datos presentes en este estudio, donde se evidenció que el perímetro corregido de pantorrilla se relacionaba con altos niveles de potencia de salto en todas sus formas (SJ, CMJ-MF y CMJML).

Los estudios de antropometría regional y atributos físicos, muestran asociaciones que involucran el peso del tren inferior, tanto con la fuerza explosiva como elástico explosivo (Ferragut et al., 2003), específicamente en este estudio, se encontró una relación directa entre el peso corporal y la potencia en el salto, lo cual, resulta ser relevante debido a que el propio peso del cuerpo proporciona una carga óptima para producir el máximo rendimiento mecánico en el salto vertical (Markovic \& Jaric, 2007), dado por la estructura corporal y los componentes musculares que generan la relación entre el peso y el desempeño del deportista (Markovic, 2007).

No obstante, Caia et al. (2013), propone que el peso del cuerpo no determina el nivel de potencia de los deportistas, sin embargo, la evidencia considera el tamaño muscular del muslo como un predictor idóneo para la generación de potencia máxima de corta duración en basquetbolista y voleibolista (Carvalho et al., 2011; Crivelin et al., 2018).

En este estudio el tamaño del muslo no presenta una relación fuerte con aspectos de desempeño físico. Contrastando análisis de antropometría regional, De Sousa et al. (2017), mostraron en basquetbolista que la variable más importante para determinar el rendimiento en el SJ y CMJ-MF es la baja cantidad de masa grasa de tronco (relación inversa, $r_{\mathrm{SJ}}=0.7 \mathrm{y} r_{\mathrm{CMJ}-\mathrm{MF}}=0.7$ ).

Por su parte, existen relaciones significativas entre los valores de potencia con los de perímetro corregido de pantorrilla, al igual que en el estudio de Bahamondes-Ávila et al. (2018), donde se evaluaron deportistas juveniles que presentaban características antropométricas de pantorrillas asociadas a la potencia de salto $(r=$ 
0.6). Al contrastar estos datos con muestra del presente estudio, se observa que existe un mayor desarrollo de la potencia y de las variables antropométricas por parte de deportistas juveniles del reciente estudio.

En torno a las medidas de pantorrilla, circunferencia y masa grasa, resultan ser variables a considerar en la ejecución del salto vertical (Rendón et al., 2017). Hermassi et al. (2019), sugiere que el volumen muscular de pantorrilla puede generar un mayor rendimiento en parámetros de fuerza en jugadores de balón mano, al igual que en el presente estudio, donde se evidencia la relación que existe entre el perímetro corregido de pantorrilla y los valores elevados de potencia.

En el estudio de Fernández-Gamboa et al. (2017), se muestra de la misma forma que los deportistas de mejor clasificación poseen un mayor desarrollo de los niveles de potencia y de circunferencia de pantorrilla. Esto puede ser debido a que una mayor área de sección transversal genera más fuerza, y a su vez, una eficiencia en el ciclo de estiramiento acortamiento, permitiendo al tendón transferir más fuerza y generar un movimiento de mayor desempeño (Alegre et al., 2016). Si bien, esto no se comprueba en el reciente estudio, se puede entender como una futura línea de investigación.

Por su parte Chelly et al. (2009) evidenció los efectos del entrenamiento de la fuerza en el desarrollo del ASMM, donde se observó que el grupo experimental tenía un mejor desempeño de la prueba de SJ $(31.5 \pm 4 \mathrm{~cm})$ y una mayor ASMM $(162 \pm 13 \mathrm{~cm} 2)$, en comparación con el grupo control, lo cual evidencia la relación directa entre el desempeño de la potencia y el ASMM (Chelly et al., 2010), al igual que los resultados del presente estudio, donde se muestra una relación moderada entre estas variables $(r \approx 0.45)$.

Finalmente, el PCP resulta tener mayor correlación con la potencia de salto, debido a su influencia en la articulación de tobillo, el cual, actúa en la transmisión de fuerza de los músculos de miembro inferior hacia el suelo (Finni, Komi \& Lepola, 2000). Por tanto, establecer relaciones entre estos aspectos puede ser un método fiable para determinar características de los deportistas en torno a la diciplina que puedan desarrollar.

\section{ConClusión}

Los principales resultados establecieron una relación entre la potencia de salto y los parámetros antropométricos de PCP, lo que permite generar una correspondencia entre estas evaluaciones, y a su vez, vincularlas con indicadores de desempeño. Junto con esto, los datos del presente estudio permiten delimitar las características de los entrenamientos de potencia y proyectar valores antropométricos de los deportistas.

Finalmente, se recomienda profundizar en el tema, a partir de la creación de investigaciones donde se midan otros tipos de variables, de las cuales, se pueda obtener mayor información sobre el desarrollo de los atletas.

\section{ReFERENCIAS}

Alegre, L. M., Hasler, M., Wenger, S., Nachbauer, W., \& Csapo, R. (2016). Does knee joint cooling change in vivo patellar tendon mechanical properties? European journal of applied physiology, 116(10), 1921-1929. https://do i.org/10.1007/s00421-016-3444-5

Al-Gindan, Y. Y., Hankey, C. R., Leslie, W., Govan, L., \& Lean, M. E. (2014). Predicting muscle mass from anthropometry using magnetic resonance imaging as reference: a systematic review. Nutrition reviews, 72(2), 113-126. https://doi.org/10.1111/nure.12096

Apostolidis, N., Nassis, G. P., Bolatoglou, T., \& Geladas, N. D. (2004). Physiological and technical characteristics of elite young basketball players. Journal of Sports Medicine and Physical Fitness, 44(2), 157. PMID: 15470313.

Bahamondes-Ávila, C., Cárcamo-Oyarzún, J., Aedo-Muñoz, E., \& Rosas-Mancilla, M. (2018). Relación entre indicadores antropométricos regionales de masa muscular y potencia de extremidades inferiores en deportistas juveniles de proyección. Revista Brasileira de Ciências do Esporte, 40(3), 295-301. https://doi.org/10.1016/j.rb ce.2018.02.002 
MHSALUD, ISSN: 1659-097X, 18(2), Julio-DiCiEMbre, 2021, pP 1-11 Benavides-Roca, Salazar Orellana, Díaz Coria

Berral de la Rosa, F.J., Rodríguez-Bies, E.C., Berral de la Rosa, C.J., Rojano Ortega, D., y Lara Padilla, E. (2010). Comparación de Ecuaciones antropométricas para evaluar la masa muscular en jugadores de badminton. International Journal of Morphology, 28(3), 803-810. http://dx.doi.org/10.4067/S0717-95022010000300022

Bosco C. (1994). La valoración de la fuerza por el test de Bosco. Paidotribo.

Bosco, C., Komi, P. V., Tihanyi, J., Fekete, G., \& Apor, P. (1983). Mechanical power test and fiber composition of human leg extensor muscles. European journal of applied physiology and occupational physiology, 51, 129-135. h ttps://doi.org/10.1007/BF00952545

Caia, J., Doyle, T. L., \& Benson, A. C. (2013). A cross-sectional lower-body power profile of elite and subelite Australian football players. The Journal of Strength \& Conditioning Research, 27(10), 2836-2841. https://doi.o $\mathrm{rg} / 10.1519 / \mathrm{JSC} .0 \mathrm{~b} 013 \mathrm{e} 3182815743$

Carvalho, H. M., Silva, M. J. C. E., Figueiredo, A. J., Gonçalves, C. E., Philippaerts, R. M., Castagna, C., \& Malina, R. M. (2011). Predictors of maximal short-term power outputs in basketball players 14-16 years. European journal of applied physiology, 111(5), 789-796. https://doi.org/10.1007/s00421-010-1703-4

Castagna, C., Ganzetti, M., Ditroilo, M., Giovannelli, M., Rocchetti, A., \& Manzi, V. (2013). Concurrent validity of vertical jump performance assessment systems. The Journal of Strength \& Conditioning Research, 27(3), 761-768. https://doi.org/10.1519/JSC.0b013e31825dbcc5

Chelly, M. S., Fathloun, M., Cherif, N., Amar, M. B., Tabka, Z., \& Van Praagh, E. (2009). Effects of a back squat training program on leg power, jump, and sprint performances in junior soccer players. The Journal of Strength \& Conditioning Research, 23(8), 2241-2249. https://doi.org/10.1519/JSC.0b013e3181b86c40

Chelly, M. S., Ghenem, M. A., Abid, K., Hermassi, S., Tabka, Z., \& Shephard, R. J. (2010). Effects of in-season shortterm plyometric training program on leg power, jump-and sprint performance of soccer players. The Journal of Strength \& Conditioning Research, 24(10), 2670-2676. https://doi.org/10.1519/JSC.0b013e3181e2728f

Crivelin, V. X., Moreira, A., Finotti, R. L., Lopes, C. R., Ramos, M., Aoki, M. S., \& Capitani, C. D. (2018). Correlação entre altura do salto e composição corporal em atletas profissionais de voleibol. Arquivos de Ciências do Esporte, 6(1), 24-27. https://doi.org/10.17648/aces.v6n1.2362

De Campos Jr, J. C., Leporace, G., \& Souto, A. (2019). Countermovement Jump Test Performance in Different Sports Modalities. Journal of Exercise Physiology Online, 22(5), 172-183.

De Sousa, S., Cintra Filho, D. A., \& Silva, R. A. (2017). Relações entre a composição corporal fracionada e total com os saltos verticais em diferentes modalidades esportivas. Revista Brasileira de Prescrição e Fisiologia do Exercício (RBPFEX), 11(71), 944-951. http://www.rbpfex.com.br/index.php/rbpfex/article/view/1316

Di Giminiani, R., \& Scrimaglio, R. (2006). Center of gravity height calculation and average mechanical power during jump performance. Italian Journal of Sport Sciences, 13, 78-84.

Fernandez-Gamboa, I., Yanci, J., Granados, C., \& Camara, J. (2017). Comparison of anthropometry and lower limb power qualities according to different levels and ranking position of competitive surfers. The Journal of Strength \& Conditioning Research, 31(8), 2231-2237. https://doi.org/10.1519/JSC.0000000000001565

Ferragut, C., Cortadellas, J., Arteaga-Ortiz, R., y Calbet, J. A. (2003). Predicción de la altura de salto vertical. Importancia del impulso mecánico y de la masa muscular de las extremidades inferiores. European Journal of Human Movement.10,7-22.

Ferragut, C., y López, J. A. (1998). Mecanismos responsables de la potenciación de la contracción muscular concéntrica en el curso del ciclo estiramiento-acortamiento. RED: Revista de entrenamiento deportivo, 12(2), 5-8.

Ferreira, A., Enes, C., Leao, C., Goncalves, L., Clemente, F. M., Lima, R., Bezerra P., \& Camoes M. (2019). Relationship between power condition, agility, and speed performance among young roller hockey elite players. Human Movement, 20(2), 24-30. https:// doi.org/10.5114/hm.2019.79040

Finni, T., Komi, P. V., \& Lepola, V. (2000). In vivo human triceps surae and quadriceps femoris muscle function in a squat jump and counter movement jump. European journal of applied physiology, 83, 416-426. https://doi.or $\mathrm{g} / 10.1007 / \mathrm{s} 004210000289$ 
MHSALud, ISSN: 1659-097X, 18(2), Julio-Diciembre, 2021, pP 1-11

Benavides-Roca, Salazar Orellana, Díaz Coria

González-Ravé, J. M., Arija, A. \& Clemente-Suarez V. (2011). Seasonal Changes in Jump Performance and Body Composition in Women Volleyball Players.J Strength Cond Res. 25(6):1492-501. https://doi.org/10.1519/J SC.0b013e3181da77f6

Hatfield, D. L., Kraemer, W. J., Spiering, B. A., Häkkinen, K., Volek, J. S., Shimano, T., Spreuwenberg P. B., Silvestre, R., Vingren J., Fragala, M., Gómez, A., Fleck, S., Newton, R., \& Maresh C. (2006). The impact of velocity of movement on performance factors in resistance exercise. The Journal of Strength \& Conditioning Research, 20(4), 760-766. https://pubmed.ncbi.nlm.nih.gov/17194227/

Hermassi, S., Chelly, M. S., Wagner, H., Fieseler, G., Schulze, S., Delank, K. S., Shephard, R. \& Schwesig, R. (2019). Relationships between maximal strength of lower limb, anthropometric characteristics and fundamental explosive performance in handball players. Sportverletzung. Sportschaden, 33(02), 96-103. https:doi.org/10.1055/s-0043-124496

Housh, D. J., Housh, T. J., Weir, J. P., Weir, L. L., Johnson, G. O., \& Stout, J. R. (1995). Anthropometric estimation of thigh muscle cross-sectional area. Medicine \& Science in Sports \& Exercise, 27(5), 784-791. PMID: 7674885.

Jiménez-Reyes, P., y González-Badillo, J. J. (2011). Control de la carga de entrenamiento a través del CMJ en pruebas de velocidad y saltos para optimizar el rendimiento deportivo en atletismo. Cultura, Ciencia y Deporte, 6(18), 207-217. https://doi.org/10.12800/ccd.v6i18.48

Jiménez, R., Parra, G., Pérez, D., \& Grande, I. (2009). Valoración de la potencia de salto en jugadores semiprofesionales de fútbol y comparación de resultados por puestos. Kronos: la revista cientifica de actividad fisica y deporte, 8(14), 79-84. http://oa.upm.es/60478/1/INVE_MEM_2009_313446.pdf

Lara, A.J, Abián, J., Alegre, L.M., Aguado, X. (2004). Tests de salto con plataforma de fuerzas en voleibol femenino. RED: Revista de entrenamiento deportivo.18(2),11-16.

Lima, R., da Silva Athayde, M., da Silva Junior, J. N., da Silva Tavares, W., \& Detanico, D. (2017). Vertical jump performance in judo and Brazilian jiu-jitsu athletes: an approach with different training levels. Ido Movement for Culture. Journal of Martial Arts Anthropology, 17(4), 25-31. https://doi.org/10.14589/ido.17.4.5

Quiroga Maraboli, P.; Bustamante Garrido, A.; Avendaño Hernández, C.; Cáceres Guerra, S.; Urrea González, S. (2016). Aumento de altura en salto en jugadores universitarios de voleibol. Apunts. Educación física y deportes, 4(126), 64-71. https://raco.cat/index.php/ApuntsEFD/article/view/316522

Marfell-Jones, M. J., Stewart, A. D., \& De Ridder, J. H. (2012). International standards for anthropometric assessment. International Society for the Advancement of Kinanthropometry.

Markovic, G. D., Dizdar, I., Jukic, M., \& Cardinale, M. (2004). Reliability and factorial validity of squat and countermovement jump tests, Journal of Strength and Conditioning Research, 18(3), 551-555. PMID: 15320660.

Markovic, G. (2007). Does plyometric training improve vertical jump height? A meta-analytical review. British journal of sports medicine, 41(6), 349-355. http://dx.doi.org/10.1136/bjsm.2007.035113

Markovic, G., \& Jaric, S. (2007). Positive and negative loading and mechanical output in maximum vertical jumping. Medicine \& Science in Sports \& Exercise, 39(10), 1757-1764. https://doi.org/10.1249/mss.0b013e31811ece35

Moreira, A., Okano, A. H., Ronque, E. R. V., de Oliveira, P. R., de Arruda, M., Mortatti, A. L., \& de Oliveira Paes, F. (2008). A dinâmica de variáveis morfológicas e de peformance motora de jovens jogadores de basquetebol. Journal of Physical Education, 19(4), 539-548. https://doi.org/10.4025/reveducfis.v19i4.3997

Ojeda-Aravena, A. P., Azócar-Gallardo, J., Hernández-Mosqueira, C., \& Herrera-Valenzuela, T. (2020). Relación entre la prueba de agilidad específica en taekwondo (tsat), la fuerza explosiva y la velocidad lineal en 5-m atletas de taekwondo de ambos sexos. Retos, 39, 84-89. https://doi.org/10.47197/retos.v0i39.78395

Oteo, J. A., Benavente, P. y Garzón, M. (2015). Valores normativos de la fuerza de puño en la población española en edad laboral. Influencia de las variables antropométricas de la mano y el antebrazo. Revista Iberoamericana de Cirugía de la Mano, 43(02), 104-110. https://doi.org/10.1016/j.ricma.2015.09.005

Ozbar, N., Ates, S., \& Agopyan, A. (2014). The effect of 8-week plyometric training on leg power, jump and sprint performance in female soccer players. The Journal of Strength \& Conditioning Research, 28(10), 2888-2894. ht tps://doi.org/10.1519/JSC.0000000000000541 
Pasin, F., Caroli, B., Spigoni, V., Dei Cas, A., Volpi, R., Galli, C., \& Passeri, G. (2017). Performance and anthropometric characteristics of Elite Rugby Players. Acta bio-médica: Atenei Parmensis, 88(2), 172. https://doi.org/10.23750 /abm.v88i2.5221

Pereira, J. E., Quintero, J. C., Peñaranda, D. G., Santamaría-Pérez, K. N., Reyes, L. A., \& Figueroa, M. A. (2018). Relación del consumo máximo de oxígeno frente al salto horizontal en jugadores de fútbol. Movimiento Cientifico, 12(1), 39-46. https://doi.org/10.33881/2011-7191.mct.12105

Raya-González, J., Suárez-Arrones, L., Rísquez, A., y Sáez de Villarreal, E. (2018). Efectos a corto plazo de un programa de entrenamiento de sobrecarga excéntrica sobre el rendimiento físico en jugadores de fútbol de élite U-16. Retos, (33), 106-111. https://doi.org/10.47197/retos.v0i33.53547

Reggiani, C., \& Schiaffino, S. (2020). Muscle hypertrophy and muscle strength: dependent or independent variables? A provocative review. European Journal of Translational Myology, 30(3). 1-12. https://doi.org/10.4081/ejtm. 2020.9311

Rendón, P. A., Chalá, L., del Rosario, L., Hernández, J.J., Alomoto, M. R., Landeta, L. J., \& Calero, S. (2017). Influencia de la masa grasa en el salto vertical de basquetbolistas de secundaria. Revista Cubana de Investigaciones Biomédicas, 36(1), 1-12. http://scielo.sld.cu/scielo.php?script=sci_arttext\&pid=S0864-03002017000100015

Rodríguez, F. J., Almagià, A. A., \& Berral de la Rosa, F. J. (2010). Estimación de la masa muscular de los miembros apendiculares, a partir de densitometría fotónica dual (DEXA). International Journal of Morphology, 28(4), 1205-1210. http://dx.doi.org/10.4067/S0717-95022010000400034

Ross, W. D., \& Kerr, D. A. (1991). Fraccionament de la massa corporal: un nou métode per utilitzar en nutrició clínica i medicina esportiva. Apunts: medicina de l'esport, 28(109), 175-188. https://www.apunts.org/en-pdf-XX8866 58191052228

Saavedra, S. y Vergara, R. (2013). Validación y comparación de tres alfombras de saltabilidad. [Tesis de licenciatura]. Universidad Santo Tomas, Santiago de Chile.

Sayers, S. P., Harackiewicz, D. V., Harman, E. A., Frykman, P. N., Rosenstein, M. T. (1999). Cross-validation of three jump power equations. Med Sci Sport Exer, 31(4),572-577. https://doi.org/10.1097/00005768-199904000-0 0013

Schober, P., Boer, C., \& Schwarte, L. A. (2018). Correlation coefficients: appropriate use and interpretation. Anesthesia \& Analgesia, 126(5), 1763-1768. https://doi.org/10.1213/ANE.0000000000002864

Sheppard, J. M., Cronin, J. B., Gabbett, T. J., McGuigan, M. R., Etxebarria, N., \& Newton, R. U. (2008). Relative importance of strength, power, and anthropometric measures to jump performance of elite volleyball players. The Journal of Strength \& Conditioning Research, 22(3), 758-765. https://doi.org/10.1519/JSC.0b013e31816 a8440

Spigolon, D., Hartz, C. S., Junqueira, C. M., Longo, A. R., Tavares, V., Fayçal, \& Moreno, M. A. (2018). The Correlation of Anthropometric Variables and Jump Power Performance in Elite Karate Athletes. Journal of Exercise Physiology online 21(1), 139-148. https://link.gale.com/apps/doc/A535421507/AONE?u=anon cfc $5 \mathrm{e} 9 \mathrm{ec} \& \mathrm{sid}=$ googleScholar\&xid=bb41dcfb

Suchomel, T. J., Nimphius, S., Bellon, C. R., \& Stone, M. H. (2018). The importance of muscular strength: training considerations. Sports medicine, 48(4), 765-785. https://doi.org/10.1007/s40279-018-0862-z

Taylor, M. J., Cohen, D., Voss, C., \& Sandercock, G. R. (2010). Vertical jumping and leg power normative data for English school children aged 10-15 years. Journal of sports sciences, 28(8), 867-872. https://doi.org/10.1080/0 2640411003770212

Temfemo, A., Hugues, J., Chardon, K., Mandengue, S-H., Ahmaidi, S. (2009) Relationship between vertical jumping performance and anthropometric characteristics during growth in boys and girls. Eur J Pediatr, 168,457-464. h ttps://doi.org/10.1007/s00431-008-0771-5 